Classification

Physics Abstracts

68.20

\title{
STM studies of Si and C evaporation on Si (111) at RT by laser ablation
}

\author{
J. Díaz, S. Ferrer and F. Comin \\ E.S.R.F., BP-220, 38043 Grenoble Cedex, France
}

(Received 1 October, 1993; accepted 25 November, 1993)

\begin{abstract}
Résumé. - On a fait des dépôts de $\mathrm{Si}$ et $\mathrm{C}$ sur la surface de $\mathrm{Si}$ (111) sous ultra vide par évaporation par laser. Le matériel évaporé contient des ions de silicium ou de $\mathrm{C}$ avec des énergies cinétiques aussi élevées que $2 \mathrm{keV}$ et $800 \mathrm{eV}$ respectivement, donc plus élevés que dans une évaporation thermique. Images obtenues in situ par microscopie a effet tunnel d'un dépôt de 0,01 monocouches montre des défauts provoquées par les atoms évaporés sur le substrat. Les défauts consistent en vacants monoatomiques sur les sites occupés pour les nommés adatoms dans la cellule unitaire, des adatoms deplacés et des cratères.
\end{abstract}

\begin{abstract}
Silicon and carbon have been deposited on Si (111) under UHV by means of the pulsed laser evaporation technique. The evaporated species contain ions of kinetic energies as high as 2 $\mathrm{KeV}$ for $\mathrm{Si}$ and $800 \mathrm{eV}$ for $\mathrm{C}$, i.e. much higher than in thermal evaporation. STM images of about 0.01 deposited monolayers reveal that some damage is produced on the substrate. The damaged areas consist mainly in monoatomic vacancies of the so called adatoms, displaced adatoms and agglomerates of vacancies.
\end{abstract}

The laser ablation technique has proved its applicability to grow different materials on different substrates. In the high power regime (more than $10^{8} \mathrm{~W} / \mathrm{cm}^{2}$ ), pulsed (ns) laser evaporation results in the emission of a high percentage of ions with kinetic energies of several hundreds of $\mathrm{eV}$. There is also a marked anisotropy in the angular distribution of the energy and concentration of out coming particles [1]. Most of the material is emitted in the direction normal to the evaporated target surface. In this direction the energy of the ions is around $2 \mathrm{KeV}$ in the case of $\mathrm{Si}$ and $800 \mathrm{eV}$ for C. For angles around $40^{\circ}$ out of the target normal, the total number of ions and their energy is strongly reduced.

Although this technique is widely used for growing superconductor thin films and other types of layers, very little is known on the details of the deposition process such as the damage produced on the substrate, the diffusion lengths of the deposited species, etc. STM (scanning tunneling microscopy) is a suitable technique to investigate the modifications of the atomic structure of the substrate and the atomic environment when the species are deposited.

Here we report on the pulsed laser evaporation of $\mathrm{Si}$ and $\mathrm{C}$ on Si (111) $7 \times 7$ for a coverage of about $0.01 \mathrm{ML}$. The results could be compared, in the case of $\mathrm{Si}$, with previously published work on growth by thermal evaporation. Köhler et al. [2] have shown, by means of STM, that the 
deposition of $1 \mathrm{ML}$ of $\mathrm{Si}$ on $\mathrm{Si}$ (111) $7 \times 7$ at room temperature leads to the formation of Si clusters in one half of the unit cell. When deposition is done at higher temperatures $\left(300^{\circ} \mathrm{C}\right)$ one obtains ordered islands of $7 \times 7$ anchored by defects.

The silicon was deposited by means of the laser ablation technique from a silicon crystal target previously outgassed to remove the oxide layer. A graphite target was used in the case of carbon. The substrate was a Si (111) crystal $n$-doped with a resistivity of $6 \mathrm{~m} \Omega . \mathrm{cm}$. Both target and substrate were inside a UHV system with a base pressure of $2 \times 10^{-10} \mathrm{mbar}$. The substrate preparation was done by high temperature annealing $\left(1200^{\circ} \mathrm{C}\right)$ following the method described in reference [3]. Before the deposition, the LEED pattern showed a sharp $7 \times 7$ reconstruction. The corresponding STM images revealed flat terraces of up to $10^{6} \AA^{2}$ with the well known adatom ordering and a density of monoatomic vacancies of $1.5 \%$ or less.

All the STM measurements were done in situ in a UHV environment. The substrate was positioned $40^{\circ}$ off the target normal. A Nd:YAG laser delivering pulses of around $250 \mathrm{~mJ}$ at $532 \mathrm{~nm}$, with a duration of $10 \mathrm{ns,}$, was employed for the ablation process. The power laser density at the target position was estimated around $10^{10} \mathrm{~W} / \mathrm{cm}^{2}$. A previous calibration [4] gave a coverage of 0.01 ML deposited after a single shot.

Time of flight measurements were performed in a separate vacuum system to determine the kinetic energy of the evaporated $\mathrm{Si}$ and $\mathrm{C}$ ions. It was found that at $40^{\circ}$ from the target normal, the energy of the ions was around $1000 \mathrm{eV}$ for $\mathrm{Si}$ and $\sim 500 \mathrm{eV}$ for $\mathrm{C}$.

After the deposition of $\mathrm{Si}$, the original $7 \times 7$ reconstruction was recovered with a temperature flash at $700{ }^{\circ} \mathrm{C}$.

The microscope was a commercial instrument (McAllister Technical Services), modified to fit our design of the sample holder. The tip-sample approach was inertial. The images shown in what follows were made in topographic mode with voltages ranging between 1.8 and $2.2 \mathrm{~V}$ (both polarities). Typical current value was $1 \mathrm{nA}$. All the forthcoming images show raw data.

Before deposition, there were not impurities diffused to the steps and the defects were adatom vacancies and disordered triangular islands trapped within domain boundaries of the $7 \times 7$ reconstruction [5]. Such a kind of islands has already been reported for the case of $\mathrm{Ge}$ (111) [6] and they should be related to the way of heating and cooling the sample. Its density was very low. After a single laser shot (coverage of $0.01 \mathrm{ML}$ ) the LEED pattern showed the $7 \times 7$ reconstruction, but the spots were broader indicating smaller sizes of the coherently reconstructed domains. Figure 1 shows the aspect of the surface after deposition of (a) $\mathrm{Si}$ and (b) C. $7 \times 7$ reconstruction is visible among regions were adatoms have been removed. These regions are distributed randomly. In the damaged regions some adatoms are displaced from their equilibrium positions and they appear brighter in the image. For example, in figure 1c, a hole appears to be surrounded by bright atoms, as a crater. Figure $1 \mathrm{~d}$ shows clearly an adatom displaced from the $7 \times 7$ reconstruction adatom position.

No sign for diffusion of adatoms towards the substrate steps was seen. Triangular islands were sometimes observed on the substrate but no evidence was found allowing to associate these islands with the deposition process.

At a lower coverage two kind of defects clearly different from those that come from residual gas contamination were observed. The most common one consists on a bright atom on top of the adatom layer close to a single or double adatom vacancy and a displaced adatom. In few cases, clusters of five or more vacancies forming a hole of more than one monolayer in depth were found surrounded by "bright"atoms. If we assume that each defect is created by a single C atom, by examining a series of images we found that, on the average, every $\mathrm{C}$ atom seems to generate 2 adatom vacancies. At a higher coverage, defects could overlap resulting in a surface made of agglomerates of adatom vacancies, single vacancies, "bright"atoms and displaced adatoms as the images $1 \mathrm{a}$ and $1 \mathrm{~b}$ show. For such a coverage, the number of vacancies are correlated to the 
a),
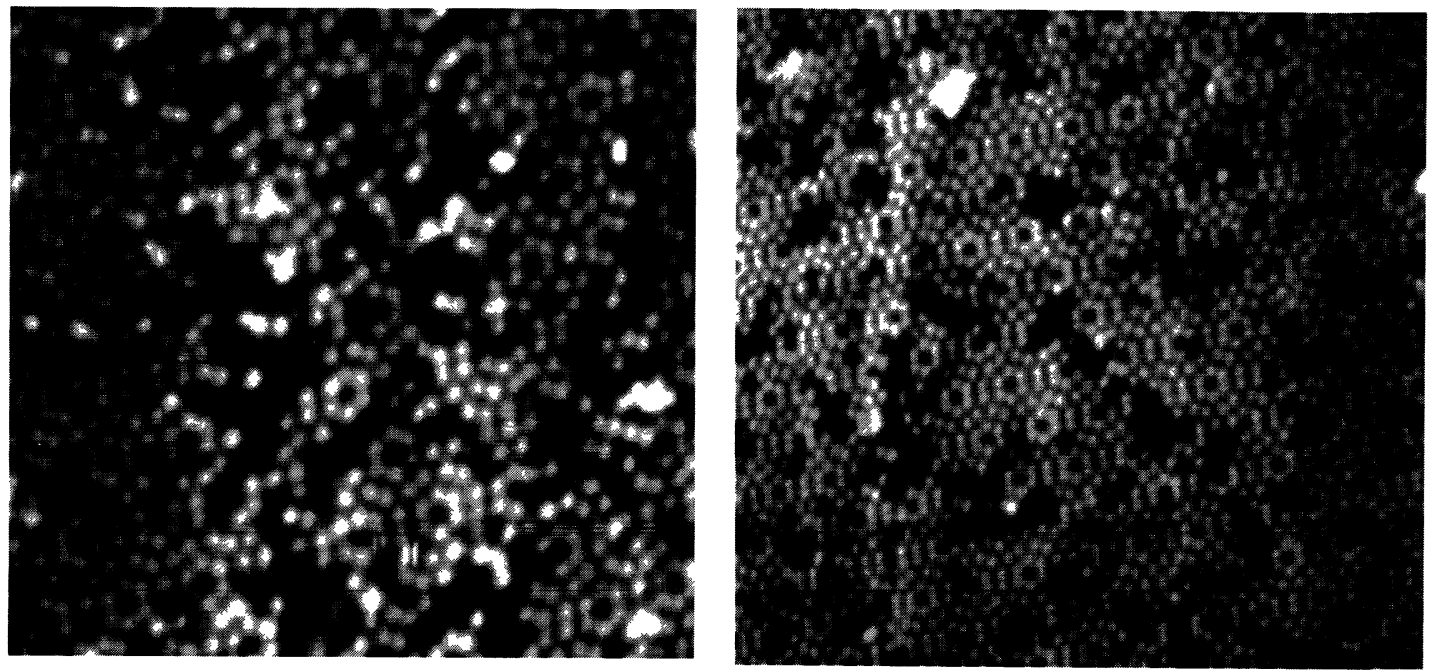

b)

c)
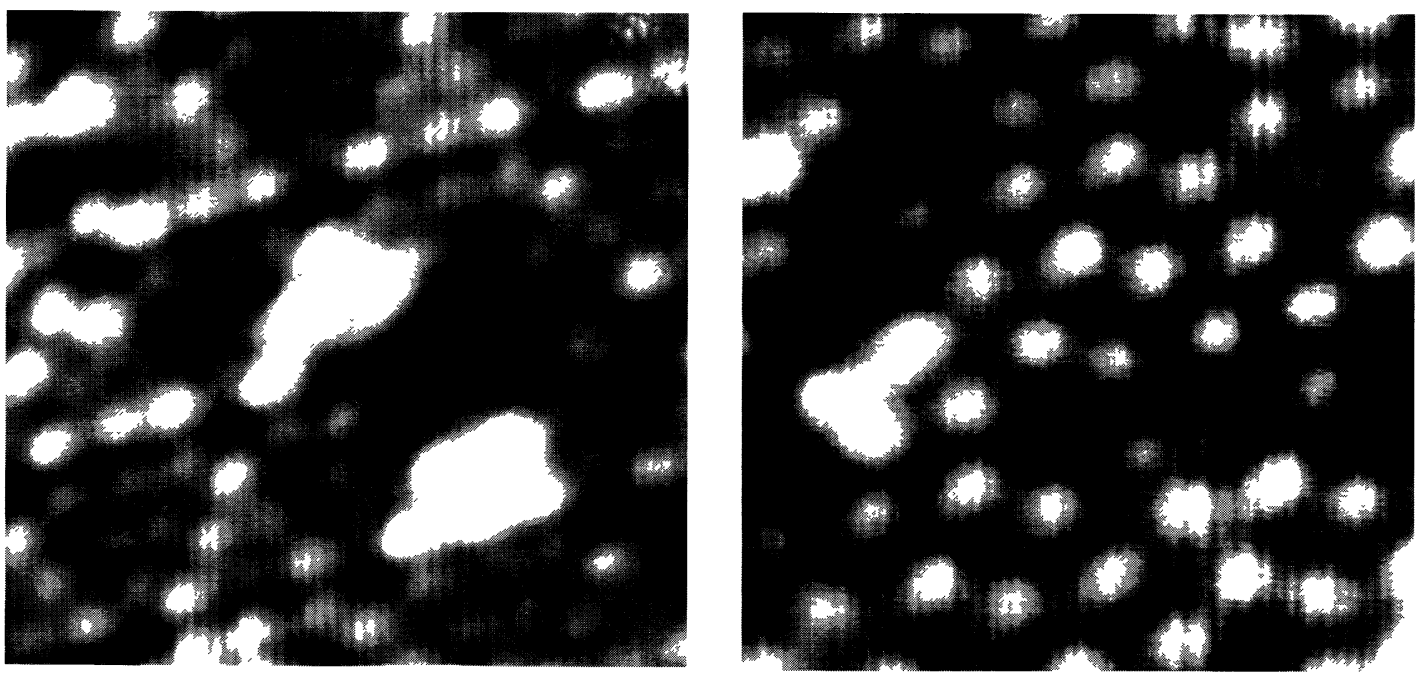

d)

Fig. 1. - Topographic images of a) $0.01 \mathrm{ML}$ of $\mathrm{Si}$ on $\mathrm{Si}(111) 7 \times 7,2000 \mathrm{mV}, 1 \mathrm{nA}, 200 \AA \times 200 \AA$; b) 0.01 ML of C on Si, $1800 \mathrm{mV}, 1 \mathrm{nA}, 300 \AA \times 300 \AA$; c) a crater. The hole is $\sim 20$ Åwide and $\sim 6 \AA$ deep. -1900 $\mathrm{mV}, 1 \mathrm{nA}, 75 \AA \times 75 \AA ;$ d) atoms beside the holes moved from adatom positions. $1900 \mathrm{mV}, 1 \mathrm{nA}, 50 \AA \times 50$ $\AA$.

calculated number of arrived $\mathrm{C}$ atoms in agreement with the ratio already mentioned. In the case of $\mathrm{Si}$, this correlation could not be established due to the lack of precise coverage measurements.

Figure 2 sketches the dangling bonds in the $7 \times 7$ reconstruction unit cell, following the DAS model [7]. The rest atoms (small circles) are in a plane below the adatoms (circles). In the operating conditions, only the adatoms were visible. They can be classified by their chemical activity: the adatoms in the inner part of the unit cell (internal adatoms), are more reactive than those beside the corner holes (external adatoms) [8, 9]. Exposing the clean surface to the residual gas of the UHV chamber for one day, the number of adatom vacancies increased sensibly. The number of vacancies of internal adatoms was twice than of the external ones which confirms their higher re- 
activity. However, it was found the same number of internal than external vacancies when silicon or carbon were evaporated by laser ablation, as it is visible by analyzing figures $1 \mathrm{a}$ and $1 \mathrm{~b}$.

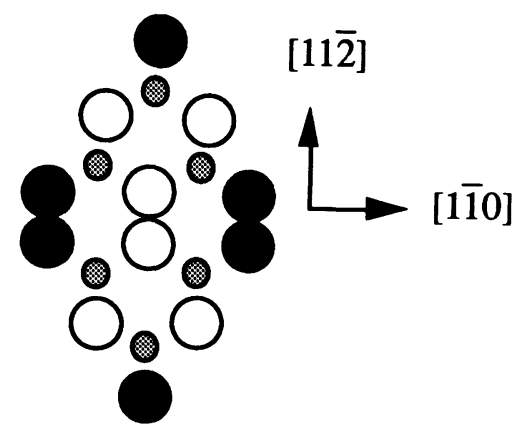

Fig. 2. - Diagram of the dangling bonds present in the unit cell of the $\mathrm{Si}(111) 7 \times 7$ reconstruction following the DAS model [5]. Full circles: external adatoms; empty circles: internal adatoms; small circles: rest atoms, they are in the plane below the adatoms.

Since an ion with kinetic energy of $15 \mathrm{eV}$ is already able to displace an adatom [10], certainly the ions from the ablation plume which have much larger energies will cause adatom displacements. This is the most likely cause for the observed defects induced by the deposition [11].

However not all the evaporated material are ions. Experiments with carbon targets under vacuum show that the ions are only $12 \%$ of the total ejected mass [12]. As the kinetic energy of the neutral atoms is not known their role on the surface growth process namely creation of vacancies or of a epitaxial layer is unclear.

In conclusion, it has been found by STM that the growth of minute amounts of both silicon and carbon causes the displacement of substrate adatoms from their equilibrium positions leaving behind multiple vacancies aggregates that appear as holes in the STM images. No doubt that these holes at the surface will slow down surface diffusion process which will lead to an increase disorder in the growth.

\section{References}

[1] Cheung J.T. and Sankur H., Crit. Rev. Solid State Mater. Sci. 15 (1988) 63.

[2] Köhler U.K., Demuth J.E. and Hamers R.J., J. Vac Sci. Technol. A7 (1989) 2860.

[3] Swartzentruber B.S., Mo X.M., Webb M.B., Lagally M.G., J. Vac. Sci. Technol. A7 (1989) 2901.

[4] Martin-Gago J.A., Comin F., Vazquez L., Bernard P. and Ferrer S., Mater. Sci. Eng. B 11 (1992) 337.

[5] Sumita I., Yokotsuka T., Tanaka H., Udagawa M., Katanabe Y., Takao M., K. Yokoyama, Appl. Phys. Lett. 57 (1991) 1313.

[6] Feenstra R.M., Slavin A.J., Held G.A. and Lutz M.A., Phys, Rev. Lett. 66 (1991) 3257.

[7] Tagayanagi K., Tanishiro Y., Takahashi M. and Takahashi S., J. Vac. Sci. Technol. A3 (1985) 1502.

[8] Wolkow R. and Avouris Ph., Phys. Rev. Lett. 60 (1988) 1049.

[9] Pelz J.P. and Koch R.H., J. Vac. Sci. Technol. B 9 (2) (1991) 775.

[10] Andersen H.H., Appl. Phys. 18 (1979) 131.

[11] Zandvliet H.J.W., Elswijk H.B., Van Loenen E.J. and Tsong I.S., Phys. Rev. B 46 (1992) 7581.

[12] Diaz J., Ferrer S., Comin F., Proceedings of the second Conference on Laser Ablation, COLA'93 (1993) 198. 